\title{
THE EXISTENCE AND STABILITY OF MULTI-DIMENSIONAL SHOCK FRONTS
}

\author{
BY ANDREW MAJDA ${ }^{1}$
}

We announce a proof of the short-time existence of a particularly interesting class of discontinuous weak solutions for the $m \times m$ system of hyperbolic conservation laws in several space dimensions,

$$
\begin{gathered}
\frac{\partial u}{\partial t}+\sum_{j=1}^{N} \frac{\partial}{\partial x_{j}} F_{j}(u)=0, \quad t>0, \\
u(x, 0)=u_{0}(x),
\end{gathered}
$$

where $x=\left(x_{1}, \ldots, x_{n}\right) \in R^{N}, u={ }^{t}\left(u_{1}, \ldots, u_{m}\right)$, and the $F_{j}(u)$ are smooth nonlinear mappings from $R^{m}$ into $R^{m}$ with $A_{j}(u)=\partial F_{j} / \partial u$ the corresponding Jacobian matrices. The important physical examples in gas dynamics, shallow water theory, and magnetofluid dynamics are all hyperbolic systems with the form in (1).

The only previous general existence results for systems of conservation laws in several space dimensions are due to Kato [2] who proved the local existence for solutions of $(1)$ when $u_{0}(x)$ is a sufficiently smooth function. For discontinuous solutions in multi-dimensions, the only previous results for gas dynamics are the explicit spherically symmetric shock fronts described in [1].

Under suitable structural assumptions, in [4], we prove the short-time existence of shock front solutions for the systems in (1). These are piecewise smooth weak solutions of (1) with the following intuitive structure: There are a smooth hypersurface, $S(t)$, defined in $(x, t)$ space for $t \geqslant 0$, noncharacteristic for (1), with space-time normal, $\left(n_{t}, n_{x}\right)$, and two smooth vectors functions, $u^{+}(x, t)$ and $u^{-}(x, t)$, defined on respective domains, $G^{+}$and $G^{-}$, on either side of this hypersurface so that

$$
\begin{aligned}
& \frac{\partial u^{+}}{\partial t}+\sum_{j=1}^{N} A_{j}\left(u^{+}\right) \frac{\partial u^{+}}{\partial x_{j}}=0 \quad \text { in } G^{+}, \\
& \frac{\partial u^{-}}{\partial t}+\sum_{j=1}^{N} A_{j}\left(u^{-}\right) \frac{\partial u^{-}}{\partial x_{j}}=0 \quad \text { in } G^{-} .
\end{aligned}
$$

Received by the editors November 6, 1980.

1980 Mathematics Subject Classification. Primary 65M10, 65M05.

1 Partially supported by N. S. F. Grant \#MCS79-02735.

() 1981 American Mathematical Society 0002-9904/81/0000-0208/\$01.75 
Furthermore, in order to define a weak solution of (1), the boundary values of $u^{+}$and $u^{-}$restricted to the hypersurface, $S(t)$, are not arbitrary and satisfy the Rankine-Hugoniot jump conditions,

$$
\left.n_{t}\left(u^{+}-u^{-}\right)\right|_{S}+\left.\sum_{j=1}^{N} n_{j}\left(F_{j}\left(u^{+}\right)-F_{j}\left(u^{-}\right)\right)\right|_{S}=0 .
$$

Since the surface, $S$, is not known in advance and must be determined as part of the solution of the problem, the equations in (2) and (3) describe a multi-dimensional nonlinear free boundary value problem for a system of quasi-linear hyperbolic equations.

We assume that the initial data in (1) is piecewise smooth with a jump discontinuity across a smooth compact hypersurface, $M_{0}$, which defines the initial position for the shock front, $S(t)$. We denote the inside of $M_{0}$ by $\Omega_{-}$and the outside of $M_{0}$ by $\Omega_{+}$with corresponding pieces of the initial data given by $u_{0}^{+}$, $u_{0}^{-}$. Necessarily, the main requirement on the initial data is that (3) is satisfied at time $t=0$; thus, there is a smooth function, $\sigma(\alpha)$, for $\alpha \in M_{0}$ so that

$$
\sigma(\alpha)\left(u_{0}^{+}(\alpha)-u_{0}^{-}(\alpha)\right)+\sum_{j=1}^{N} n_{j}(\alpha)\left(F_{j}\left(u_{0}^{+}(\alpha)\right)-F_{j}\left(u_{0}-(\alpha)\right)\right)=0
$$

for all $\alpha \in M_{0}$, where $n=\left(n_{1}(\alpha), \ldots, n_{N}(\alpha)\right)$ is the outward normal to $M_{0}$. We also require a finite number of compatibility conditions on the normal derivatives of the initial data restricted to $M_{0}$. For the physical examples, given an arbitrary $M_{0}$, a wide class of initial data can be constructed to satisfy these conditions [4].

Rather than stating the main theorem in [4] in full generality, we restrict ourselves here to a precise description for the simplest multi-dimensional physical system, the $3 \times 3$ system describing isentropic compressible flow in two space dimensions given by

$$
\frac{\partial}{\partial t}\left(\begin{array}{c}
\rho w_{1} \\
\rho w_{2} \\
\rho
\end{array}\right)+\frac{\partial}{\partial x_{1}}\left(\begin{array}{c}
\rho w_{1}^{2}+p \\
\rho w_{1} w_{2} \\
\rho w_{1}
\end{array}\right)+\frac{\partial}{\partial x_{2}}\left(\begin{array}{c}
\rho w_{1} w_{2} \\
\rho
\end{array}\right)=\left(\begin{array}{l}
0 \\
0 \\
0
\end{array}\right)
$$

where $w={ }^{t}\left(w_{1}, w_{2}\right)$ is the fluid velocity, $\rho$ is the density, and $p(\rho)$ is a welldefined function of $\rho$ with $p^{\prime}(\rho)>0$ and determined by an equation of state. Below, $H^{s}(\Omega)$ denotes the Sobolev space of order $s$ on the domain $\Omega$.

THEOREM. Assume that $M_{0}$ is an arbitrary smooth curve and the initial data, $\left(w_{0}^{-}, \rho_{0}^{-}\right)$and $\left(w_{0}^{+}, \rho_{0}^{+}\right)$belong to $H^{s}\left(\Omega_{-}\right)$and $H^{s}\left(\Omega_{+} \cap\{x|| x \mid<R\}\right)$ respectively for some fixed $s \geqslant 10$ and that $\left(w_{0}^{+}, \rho_{0}^{+}\right)$is constant for $|x|>R$. 
Also assume that there is a function $\sigma(\alpha)$ so that (4) and the compatibility conditions up to order 8 are satisfied together with the entropy conditions,

$$
\begin{gathered}
w_{0}^{+} \cdot n+p_{\rho}\left(\rho^{+}\right)< \\
\sigma(\alpha)<w_{0}^{-} \cdot n+p_{\rho}\left(\rho^{-}\right), \quad \alpha \in M_{0}, \\
\rho^{+}(\alpha)<\rho^{-}(\alpha),
\end{gathered}
$$

where $p(\rho)$ is a given convex function of $\rho$. Then there is a $C^{2}$ hypersurface $S(t)$ defined for $0 \leqslant t \leqslant T$ with $T$ sufficiently small so that a piecewise smooth shock front solution of the isentropic compressible flow equations in (5) exists with the above initial data and satisfies (2) and (3).

In particular, these results apply for shock fronts of arbitrary strength in polytropic gases where $p=A \rho^{r}, r>1$, and similar results are valid for the full Euler equations in three space dimensions. The proof has two main standard steps: (1) An appropriate linearized problem is analyzed; (2) a nonlinear iteration scheme is constructed.

The linearization of shock front solutions leads to an unusual new type of boundary value problem for hyperbolic equations which is studied in [5] by using micro-local estimates and Kreiss' symmetrizer construction [3] adapted to nonlocal boundary conditions. Several interesting new phenomena occur and these are analyzed in [5]. In particular, unlike the situation in a single space variable, in multi-dimensions, shock front solutions of the equations of ideal compressible flow have stronger stability properties than those of a scalar conservation law in several space variables; these properties are crucial for the proofs in [4].

The convergence of the nonlinear iteration scheme is described in [4]. The strong stability properties described above allow us to prove the main theorem through a classical iteration scheme so that shock fronts for the physical equations do not exhibit the "loss of derivatives" phenomena. The convergence proof depends on some unusual weighted norm estimates in space-time for the functions defined by the iteration scheme.

\section{BIBLIOG R A PHY} New York.

1. R. Courant and K. O. Friedrichs, Supersonic flow and shock waves, Springer-Verlag,

2. T. Kato, The Cauchy problem for quasi-linear symmetric hyperbolic systems, Arch. Rational Mech. Anal. 58 (1975), $181-205$.

3. H. O. Kreiss, Initial boundary value problems for hyperbolic systems, Comm. Pure Appl. Math. 23 (1970), 277-298.

4. A. Majda, The existence of multi-dimensional shock fronts, Comm. Pure Appl. Math. (to appear).

5. The stability of shock fronts-a new problem for linear hyperbolic equations, Comm. Pure Appl. Math. (to appear).

DEPARTMENT OF MATHEMATICS, 887 EVANS HALL, UNIVERSITY OF CALIFORNIA, BERKELEY, CALIFORNIA 94720 